\title{
Validation of Higher-Order Approximations and Boundary Conditions for Lossy Conducting Bodies
}

\author{
Ilya O. Sukharevsky, Member, IEEE, and Ayhan Altintas, Senior Member, IEEE
}

\begin{abstract}
The problem of high-frequency diffraction by a smooth lossy body with high conductivity is considered. In addition to the geometrical optics approximation, additional asymptotic terms are derived to take into account the curvature of the boundary and material properties. Since these higher-order terms are derived by taking into account exact boundary conditions, it is easy to learn about the limitations of impedance conditions and to determine more accurate approximate conditions. The obtained higher-order boundary conditions and their limitations are numerically validated by solving Muller's second-kind integral equations.
\end{abstract}

Index Terms-Asymptotic diffraction theory, boundary value problems, conducting materials, higher-order boundary conditions, impedance boundary conditions (IBCs).

\section{INTRODUCTION}

$\mathbf{I}$ MPEDANCE boundary conditions (IBCs) formulated by Leontovich [1] laid the foundation for using and deriving approximate conditions in electromagnetics. Such conditions can be derived in different ways, in particular, by using integral equation formulation and by selection of a small parameter [2], [3]. Typically, skin-depth is taken as the small parameter [4]. The variety of approximate boundary conditions is summarized in the fundamental book of Senior and Volakis [5]. Finding the limits of applicability of the IBC was the topic of several studies. For instance, it was shown in [6] that the IBC do not describe field behavior correctly for lossless, "pure" dielectric smooth bodies. A few well-known approaches to the derivation of approximate boundary conditions were compared to exact solutions in the comprehensive treatment of the surface impedance concept [7].

The method of getting the higher-order solutions, discussed in this paper, is based on combination of ray optics outside of the scatterer with the small parameter expansions of the fields inside the skin-layer of a conductor. Initially it was developed for problems of diffraction by thin [8], [9] and thick [10]-[12] (in terms of the wavelength) curved inhomogeneous dielectric layers, by 2-D and 3-D formulations. It was also used for diffraction by a

Manuscript received August 15, 2013; revised May 12, 2014; accepted June 11, 2014. Date of publication June 24, 2014; date of current version September 01, 2014.

I. O. Sukharevsky is with the Communication and Spectrum Management Research Center (ISYAM), Bilkent University, 06800 Bilkent, Ankara, Turkey (e-mail: i.sukharevsky@gmail.com).

A. Altintas is with the Department of Electrical and Electronics Engineering and the Communication and Spectrum Management Research Center (ISYAM), Bilkent University, 06800 Bilkent, Ankara, Turkey, and also with the Faculty of Engineering and Natural Sciences, Abdullah Gul University, 38039 Kayseri, Turkey.

Digital Object Identifier 10.1109/TAP.2014.2331991 curved dielectric layer backed by a perfectly conducting surface [13]. The heuristic assumptions about asymptotic expansions used in [8]-[13] matched the exact solutions of canonical problems (e.g., see [14]). Higher-order terms of the expansion allow estimating the influence of the surface curvature, the curvature of the initial wave front and the nonequidistancy of boundaries to the scattered field [9], i.e., the factors, which are not taken into account by approximation of the curved layer at the reflection point by a tangent plane.

The application of this method to good conductors and absorbers allows obtaining new results. Maxwell's equations, written in boundary-layer coordinates, were solved asymptotically, and each term of asymptotic expansions was obtained through the exact boundary conditions. The application of the method discussed to the skin-effect problem [15]-[17], allows finding subsequent terms of asymptotic expansions by solving 1-D boundary problems resulting from Maxwell's differential equations. The well-known Rytov's treatment [18] can be considered as a particular case of this approach. The asymptotic solutions for the problem of diffraction by conducting bodies and resulting curvature-corrected formulae for the quantity of heat emitted by the skin-layer in 2-D were briefly reported in [15], and the 3-D study of the problem was presented in [16], [17].

Impedance-type boundary conditions are often used for deriving a system of integral equations [19], [20] to be solved then numerically. As a by-product of the asymptotic analyses [15], [17], the simple first-type higher-order conditions can be obtained, and this allows considering diffraction by a conducting body, as the Dirichlet boundary problem. Needless to say, it is much more suitable for computations.

In this paper we give a detailed description of the method in 2-D, and discuss its limitations and applicability by comparing with exact numerical solutions of the problem.

\section{PROBlem Formulation}

A conducting body $G$ with complex electric permittivity $\varepsilon$, magnetic permeability $\mu$ and conductivity $\sigma$ is illuminated in free space by a plane electromagnetic wave

$$
\vec{E}^{i}(\vec{x})=\vec{ह}^{i} e^{i k_{0} \Phi(\vec{x})}, \quad \vec{H}^{i}(\vec{x})=\overrightarrow{\mathscr{C}}^{i} e^{i k_{0} \Phi(\vec{x})}
$$

where $\vec{\nabla} \Phi=\hat{l}^{i}$, with $\left|\hat{l}^{i}\right|=1$, and $k_{0}=\omega / c$, the wave number. Throughout the paper, time-factor $e^{-i \omega t}$ is assumed and suppressed. We are also using the Gaussian unit system for the sake of continuity of [8]-[12].

High-frequency formulation of diffraction by good conductors implies that $\mu \sigma / \omega \gg 1$, while $\omega \rightarrow \infty$. However, since the magnitude of $\sigma$ may vary significantly according to the type of 
the lossy material, this fact is to be taken into account to satisfy the cited condition, when checking the applicability of the IBC and deriving the solution. This reason was the initial point for the derivation of our theory.

Let $\hat{N}$ be the internal unit normal to the boundary of $G$ defined by $x(s, 0)$ with $s$ is the boundary contour coordinate; and $S$ is the part of this boundary illuminated by the incident wave: $\left(\hat{l}^{i} \cdot \vec{x}(s, 0)\right)>0$.

Let us define the following dimensionless small parameters:

$$
\zeta=\sqrt{\frac{\omega}{2 \pi \mu \sigma}}, \quad \eta=\frac{\kappa_{0}}{k_{0}}
$$

whose product is $\xi \equiv \eta \zeta=\kappa_{0} \delta$. Here $\kappa_{0}=1 / R_{0}$ is the maximal curvature of $S$, and $\delta=c / \sqrt{2 \pi \omega \mu \sigma}=\zeta / k_{0}$ is the skin-layer thickness that is assumed to be small compared both to the wavelength $(\zeta \ll 1)$ and the minimal curvature radius $R_{0}(\eta \zeta \ll 1)$. We also assume that $|\mu|$ and $\operatorname{Re} \varepsilon=\varepsilon_{1}$ are not big.

As the complex dielectric permittivity is $\varepsilon=\varepsilon_{1}+4 \pi i \sigma / \omega$, we obtain

$$
\varepsilon=\varepsilon_{1}+2 i /\left(\mu \zeta^{2}\right)
$$

resulting $\operatorname{Im} \varepsilon \gg 1$.

For high-frequency diffraction by the conducting body $\eta \ll$ 1 , and we have two independent small parameters: $\eta$ and $\zeta$. Therefore, asymptotic solution of this problem is to be found for a certain ratio of smallness order $\eta$ and $\zeta$. S. M. Rytov's analysis can be interpreted as the solution of this problem for $\eta \sim 1$, since the field expansions of [18] can be written as expansions over dimensionless small parameter $\zeta$, in which connection $\eta \zeta \sim \zeta$.

Let parameters $\eta$ and $\zeta$, defined by (2), be related as

$$
\eta=\alpha \zeta^{p / q}
$$

where $\alpha \sim 1$, and $p, q$ are natural mutually prime numbers. As is obvious, in this case $\xi=\alpha \zeta^{(p+q) / q}$.

In real problems, the relation (4) is dependent on the wavelength $\lambda_{0}=2 \pi / k_{0}$, minimal curvature radius $R_{0}=1 / \kappa_{0}$ and physical parameters of the medium $(\sigma$ and $\mu$ ).

The case of $p \leq q$ is more typical for diffraction by nonferromagnetic metallic bodies, when $\zeta$ is in the order of $10^{-3}$ to $10^{-4}, \eta=10^{-1}$ to $10^{-3}$ for the wavelength range of $\lambda=10^{-1}$ to $10 \mathrm{~cm}$, and the case of $p>q$ may refer, for example, to diffraction by biological tissues at $\lambda \sim 1 \mathrm{~cm} \mathrm{[21],} \mathrm{or} \mathrm{to} \mathrm{scat-}$ tering by a wet soil in the meter wave-range [22]. Therefore, both cases are of our interest.

\section{BASIC EQUATIONS AND EXACT BOUNDARY CONDITIONS}

Let us represent the electromagnetic field in the outer domain $G_{1}$ (Fig. 1) as the sum of incident and reflected fields, and suppose the reflected field $\left(\vec{E}^{r}, \vec{H}^{r}\right)$ to have a ray expression, as in [15]-[17]

$$
\vec{E}^{r}=\overrightarrow{\mathscr{E}}^{r}(\vec{x}) e^{i k_{0} \Psi(\vec{x})}, \quad \vec{H}^{r}=\overrightarrow{\mathscr{C}}^{r}(\vec{x}) e^{i k_{0} \Psi(\vec{x})}
$$

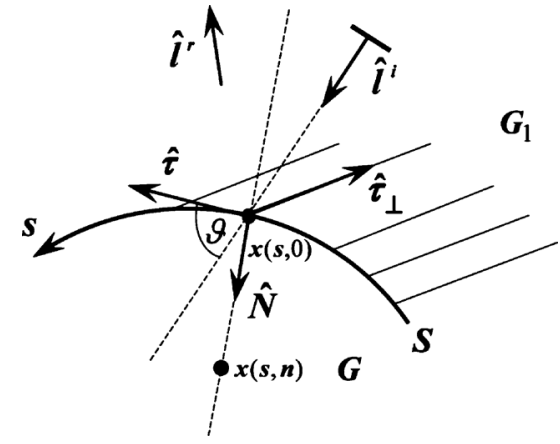

Fig. 1. Problem geometry.

and the direction of propagation of reflected rays is $\hat{l}^{r}=\vec{\nabla} \Psi$.

Inside the skin layer, let us introduce curvilinear coordinates $(s, n)$, where $n$ is the distance from $S$ measured along the internal unit normal $\hat{N}$. In addition to coordinates $(s, n)$, we also use in $G$ the coordinates $(\bar{s}, \nu)$, where $\bar{s}=\kappa_{0} s, \nu=n / \delta(0 \leq$ $\nu \leq 1$ ), and assume

$$
\vec{E}=\vec{E}(s, n) e^{i k_{0} \Phi(s, 0)}, \quad \vec{H}=\ddot{\mathscr{C}}(s, n) e^{i k_{0} \Phi(s, 0)}
$$

where $\vec{E}, \vec{H}$ are the fields inside the skin-layer.

\section{A. Derivation of Basic Equations}

For definiteness, consider E-polarization, i.e., $\vec{\varepsilon}=$ $U(s, n) \hat{\tau}_{\perp}, \overrightarrow{\mathscr{H}}=V(s, n) \hat{\tau}$, where $U, V$ are some functions, $\hat{\tau}_{\perp}=(\hat{N} \times \hat{\tau})=$ const, and $\hat{\tau}=\hat{\tau}(s)$ is a unit tangent to $S$ (Fig. 1).

The solution of our diffraction problem for 2-D fields in (6) must satisfy Helmholtz equation [23]

$$
\nabla^{2}\left(e^{i k_{0} \Phi(s, 0)} U\right)+k_{0}^{2} \varepsilon \mu e^{i k_{0} \Phi(s, 0)} U=0 .
$$

By using obvious relation $\partial \hat{N} / \partial s=\kappa \hat{\tau}$, we write the 2-D Laplasian as

$$
\nabla^{2}=\frac{1}{(1+\kappa n)^{2}} \frac{\partial^{2}}{\partial s^{2}}+\frac{\kappa}{1+\kappa n} \frac{\partial}{\partial n}+\frac{\partial^{2}}{\partial n^{2}}
$$

where $\kappa=\kappa(s)$ is a curvature of $S$ at $\vec{x}(s, 0)$.

Upon rewriting (7) in dimensionless coordinates $(\bar{s}, \nu)$, we obtain

$$
\frac{\partial^{2} U}{\partial \nu^{2}}+\zeta^{2}[\varepsilon \mu U-Z(Z U)]+\frac{\bar{\kappa} \xi}{1+\bar{\kappa} \xi \nu} \frac{\partial U}{\partial \nu}=0
$$

where $Z=(1) /(1+\bar{\kappa} \xi \nu)(\cos \vartheta-i \eta(\partial) /(\partial \bar{s})), \vartheta$ is the angle between $\hat{\tau}$ and $\hat{l}^{i}$ (i.e., $\cos \vartheta=\partial \Phi(s, 0) / d s=$ $\left.\hat{\tau}(s) \cdot \hat{l}^{i}(s, 0)\right), \bar{\kappa}=\kappa / \kappa_{0}$ is the normalized curvature, and $\beta=\mu$. Note that the angle of incidence is the complimentary angle of $\vartheta$ (Fig. 1).

The relation between electric and magnetic fields in $G$ and $G_{1}$ can be obtained easily from one of Maxwell's equations $\left(i k_{0} \mu \vec{H}=\vec{\nabla} \times \vec{E}\right)$

$$
i k_{0} \mu(\hat{N} \times \vec{H})=-\partial \vec{E} / \partial n
$$

since $\vec{E} \cdot \hat{N}=0$. 
By substituting ray representations (6) into (10), we derive the next relation for the fields in $G$ in coordinates $(\bar{s}, \nu)$

where $\beta=\mu$.

$$
V=-\frac{1}{i \zeta \beta} \frac{\partial U}{\partial \nu}
$$

\section{B. Exact Boundary Conditions in a Special Form}

Assume that $\varepsilon=\mu=1(10)$ and, thus, suppose $\left(\vec{E}^{t}, \vec{H}^{t}\right)$ to be the field in $G_{1}$

$$
\vec{E}^{t}=\vec{E}^{i}+\vec{E}^{r}, \quad \vec{H}^{t}=\vec{H}^{i}+\vec{H}^{r}
$$

where $\vec{E}^{i}, \vec{E}^{r}, \vec{H}^{i}, \vec{H}^{r}$ are defined by (1), (5), (6). Since $\partial \Phi / \partial n=-\partial \Psi / \partial n$, after limiting transition from $G_{1}$ to $S$, by taking projection of left and right-hand parts of (10) onto $\hat{\tau}_{\perp}$, we obtain

$\left.i k_{0}\left(V^{i}+V^{r}\right)\right|_{S}=-\partial\left(U^{i}+U^{r}\right) / \partial n-i k_{0}\left(U^{i}-U^{r}\right) \partial \Phi / \partial n$

or

$$
\begin{aligned}
\left.i k_{0}\left(V^{i}+V^{r}\right)\right|_{S}= & -\partial\left(U^{i}+U^{r}\right) / \partial n-2 i k_{0} U^{i} \partial \Phi / \partial n \\
& +i k_{0}\left(U^{i}+U^{r}\right) \partial \Phi / \partial n
\end{aligned}
$$

where $U^{i}=\vec{\delta}^{i} \cdot \hat{\tau}_{\perp}, U^{r}=\vec{\delta}^{r} \cdot \hat{\tau}_{\perp}$.

Since $\partial U^{i} /\left.\partial n\right|_{S} \equiv 0$ for the plane wave (1), and $V^{i}+$ $\left.V^{r}\right|_{S}=V, U^{i}+\left.U^{r}\right|_{S}=U$, (14) takes form

$$
i k_{0} V=-\partial U^{r} / \partial n-2 i k_{0} U^{i} \partial \Phi / \partial n+i k_{0} U \partial \Phi / \partial n .
$$

Substituting (11) into (15), taking into account (4) and that $\partial \Phi / \partial n=\left(\hat{l}^{i} \cdot \hat{N}\right)=\sin \vartheta$, we write the exact boundary condition in dimensionless coordinates $(\bar{s}, \nu)$

$$
\begin{array}{r}
\frac{1}{\mu} \frac{\partial U}{\partial \nu}+i \zeta \sin \vartheta U=2 i \zeta \sin \vartheta U^{i}+\frac{\alpha \zeta^{1+p / q}}{\kappa_{0}} \frac{\partial U^{r}}{\partial n} \\
(\nu=0) .
\end{array}
$$

The second-order boundary value problem (9), (16) is wellsuited for asymptotic solution.

The derivation of formulas in the case of $\mathrm{H}$-polarization is entirely analogous. In this respect, $\beta=\varepsilon$ in (11), and $U^{i}, U, V, U^{r}$ in (9), (11) and (15) have the following meaning:

$$
U^{i}=\overrightarrow{\mathscr{C}}^{i} \cdot \hat{\tau}_{\perp}, U^{r}=\overrightarrow{\mathscr{K}}^{r} \cdot \hat{\tau}_{\perp}, U=\overrightarrow{\mathscr{C}} \cdot \hat{\tau}_{\perp}, V=-\overrightarrow{\mathscr{E}} \cdot \hat{\tau}
$$

and, by taking into account (3), we derive from (15) the boundary condition analogous to (16), at $\nu=0$

$$
\frac{\mu \zeta}{2-i \varepsilon_{1} \mu \zeta^{2}} \frac{\partial U}{\partial \nu}-\sin \vartheta U=-2 \sin \vartheta U^{i}+\frac{i \alpha \zeta^{p / q}}{\kappa_{0}} \frac{\partial U^{r}}{\partial n}
$$

\section{Asymptotic Solution of the Problem}

By substituting expression for $Z$ into (9) and taking into account (3), (4), we obtain

$$
\frac{\partial U^{2}}{\partial \nu^{2}}+2 i U=-\zeta^{2}\left(\varepsilon_{1} \mu-\cos ^{2} \vartheta\right) U-\alpha \bar{\kappa} \zeta^{1+\frac{p}{q}} \frac{\partial U}{\partial \nu}+\zeta^{2+\frac{p}{q}} \mathscr{L} U
$$

where $\mathscr{L}$ is some differential operator over $\nu$ and $\bar{s}$. Since the summand comprising the operator $\mathscr{L}$ represents higher-order terms neglected in our consideration, we do not give explicit representation of it.

We now proceed to find functions $U, V, U^{r}$ in the form of expansions

$$
\begin{aligned}
U & \sim \sum_{m=0}^{\infty} U_{m}(s, \nu) \zeta^{m / q} \\
V & \sim \sum_{m=0}^{\infty} V_{m}(s, \nu) \zeta^{m / q}, \quad(0<\nu<1) ; \\
U^{r} & \sim \sum_{m=0}^{\infty} U_{m}^{r}(s, \nu) \zeta^{m / q},(n<0) .
\end{aligned}
$$

Boundary condition on $S\left(U^{i}+U^{r}=U\right)$ generates the system of equations on $S$

$$
U^{i}+U_{0}^{r}=U_{0}, \quad U_{m}^{r}=U_{m}(m=1,2,3, \ldots)
$$

where $U_{0}$ is the principal term of (20), and $U_{m}$ are subsequent ones.

Beside that, we impose the physically natural condition of attenuation inside $G$ on functions $U_{m}, V_{m}$, implying

$$
\lim _{\nu \rightarrow+\infty} U_{m}(s, \nu)=\lim _{\nu \rightarrow+\infty} V_{m}(s, \nu)=0 .
$$

To determine $\partial U^{r} / \partial n$ in (16), we use one more important boundary relation on $S$, resulting from (21) and the "ray" Helmholtz equation $\left(\nabla^{2}+k_{0}^{2}\right)\left(e^{i k_{0} \Psi} U^{r}\right)=0$, which can be written as

$$
2 \partial U^{r} / \partial l^{r}+\nabla^{2} \Psi U^{r}+\nabla^{2} U^{r} /\left(i k_{0}\right)=0 .
$$

By substituting (20) into (23), we obtain the following relations:

$$
\begin{aligned}
2 \partial U_{0}^{r} / \partial l^{r}+\nabla^{2} \Psi U_{0}^{r} & =0 \\
\kappa_{0}\left[2 \partial U_{1}^{r} / \partial l^{r}+\nabla^{2} \Psi U_{1}^{r}\right]-i \nabla^{2} U_{0}^{r} & =0
\end{aligned}
$$

and further relations for the neglected higher-order terms.

For the plane-wave excitation, $\nabla^{2} \Psi=-2 \kappa / \sin \vartheta$, and $\partial U^{i} / \partial \bar{s} \equiv 0$. Therefore, taking into account $\partial U_{0}^{r} / \partial l^{r}=$ $\cos \vartheta \partial U_{0}^{r} / \partial s-\sin \vartheta \partial U_{0}^{r} / \partial n$, we derive from (24)

$$
\begin{aligned}
\frac{1}{\kappa_{0}} \frac{\partial U_{0}^{r}}{\partial n} & =\cot \vartheta \frac{\partial U_{0}^{r}}{\partial \bar{s}}-\frac{\bar{\kappa}}{\sin ^{2} \vartheta} U_{0}^{r} \\
& =\cot \vartheta \frac{\partial U_{0}}{\partial \bar{s}}-\frac{\bar{\kappa}}{\sin ^{2} \vartheta}\left(U_{0}-U^{i}\right) .
\end{aligned}
$$

As it will be shown later, $U_{0} \equiv 0$; hence, $\nabla^{2} U_{0}^{r}=-\nabla^{2} U^{i} \equiv$ 0 , and (25) takes the same view, as (24).

From (19), (11), and (16) (or (19), (11) and (18), respectively), it is easy to derive now the sequence of boundary problems for coefficients of (20) in both $E$ - and $H$-polarization cases. 


\section{E-POLARIZATION}

By substituting (20) into (19) and (16), and enforcing equality between the expansion coefficients of the same order of $\zeta$, we obtain for $0 \leq m<q$

$$
\left\{\begin{array}{l}
\partial^{2} U_{m} / \partial \nu^{2}+2 i U_{m}=0, \quad(\nu>0) \\
\partial U_{m} /\left.\partial \nu\right|_{\nu=0}=0
\end{array}\right.
$$

Hence, using (22), we obtain, that

$$
U_{0} \equiv U_{1} \equiv \cdots \equiv U_{q-1} \equiv 0 .
$$

The other nonzero term is derived from the next boundary value problem

$$
\left\{\begin{array}{l}
\partial^{2} U_{q} / \partial \nu^{2}+2 i U_{q}=0, \quad(\nu>0) \\
\frac{1}{\mu} \frac{\partial U_{q}}{\partial \nu}+i \sin \vartheta U_{0}=2 i \sin \vartheta U^{i}, \quad(\nu=0) .
\end{array}\right.
$$

Hence

$$
U_{q}(s, \nu)=2 U^{i} \sin \vartheta i \mu e^{(i-1) \nu} /(i-1)
$$

and from (11), we get

$$
V_{0}(s, \nu)=-\frac{1}{i \mu} \frac{\partial U_{q}(s, \nu)}{\partial \nu}=-2 U^{i} \sin \vartheta e^{(i-1) \nu} .
$$

This implies that in the main asymptotic approximation $(U \sim$ $\left.\zeta U_{q}, V \sim V_{0}\right)$, the impedance Leontovich condition is fulfilled on $S(\nu=0)$

$$
U \sim-w V
$$

where $w=(1-i) \mu \zeta / 2$ is the impedance.

Finding the higher-order asymptotic approximations will be carried out for certain cases of $p<q, p=q$ and $p>q$.

\section{A. Case $p<q$.}

Using (19) we get for $0<j<p$

$$
\partial^{2} U_{q+j} / \partial \nu^{2}+2 i U_{q+j}=0, \quad(\nu>0)
$$

and, for the same values of $j$, it follows from (16) and (28) that $\partial U_{q+j} /\left.\partial \nu\right|_{\nu=0}=0$.

Consequently, $U_{q+1} \equiv \cdots \equiv U_{q+p-1} \equiv 0$.

For the next term, for $\nu>0$

$$
\left\{\begin{array}{l}
\partial^{2} U_{q+p} / \partial \nu^{2}+2 i U_{q+p}=-\alpha \bar{\kappa} \partial U_{0} / \partial \nu=0,(\nu>0) \\
\frac{1}{\mu} \frac{\partial U_{q+p}}{\partial \nu}+i \sin \vartheta U_{p}=\frac{\alpha}{\kappa_{0}} \frac{\partial U_{0}^{r}}{\partial n},(\nu=0)
\end{array}\right.
$$

or, in consequence of (26) and (28): $\partial U_{q+p} /\left.\partial \nu\right|_{\nu=0}=$ $\alpha \mu \bar{\kappa} U^{i} / \sin ^{2} \vartheta$.

The solution of this system is

$$
U_{q+p}(s, \nu)=\alpha \mu \bar{\kappa} U^{i} e^{(i-1) \nu} /\left[(i-1) \sin ^{2} \vartheta\right] .
$$

Let us now proceed to find $V_{m}$. From (11) and (28), we see that

$$
\begin{aligned}
& V_{1} \equiv \cdots \equiv V_{p-1} \equiv 0 \\
& V_{p}(s, \nu)=-\frac{1}{i \mu} \frac{\partial U_{q+p}(s, \nu)}{\partial \nu}=\frac{i \alpha \bar{\kappa} U^{i}}{\sin ^{2} \vartheta} e^{(i-1) \nu} .
\end{aligned}
$$

Therefore

$$
\begin{aligned}
U & \sim \zeta\left[U_{q}(s, \nu)+\zeta^{p / q} U_{q+p}(s, \nu)\right] \\
V & \sim V_{0}(s, \nu)+\zeta^{p / q} V_{p}(s, \nu) .
\end{aligned}
$$

Note that the second asymptotic terms of (38) are dependent on curvature $\bar{\kappa}$ of the boundary $S$. By putting together boundary values of (38), we find that for $\nu=0$ impedance condition (32) is still fulfilled. This yields that in the considered case of $p<q$, which is typical for diffraction by metallic body, Leontovich boundary condition describes not only the leading asymptotic term, but also a curvature-corrected term.

At the same time, the obtained results allow to reduce the problem of finding diffracted field in $G_{1}$ not to the impedancetype boundary problem, but to Dirichlet's boundary problem on $S$

$$
\begin{aligned}
U^{r} & =-U^{i}+\zeta\left[U_{q}+\zeta^{p / q} U_{p+q}+o\left(\zeta^{p / q}\right)\right] \\
& =-U^{i}\left[1-w\left(2 \sin \vartheta-i \alpha \bar{\kappa} \zeta^{p / q} / \sin ^{2} \vartheta+o\left(\zeta^{p / q}\right)\right)\right] .
\end{aligned}
$$

It can be easily checked that the asymptotic approximation comprising the term of $\zeta^{2+p / q}$ order does not satisfy Leontovich condition anymore.

For instance, by deriving higher-order asymptotic terms for $p=1, q=2$, one can show that, for the fifth-term approximation, (32) changes into

$$
U+w V \sim-(1+i) a(s) \zeta^{5 / 2} / 2
$$

where $a(s)=\alpha \bar{\kappa} i \mu \sin \vartheta U^{i} /(i-1)$.

\section{B. Case $p=q=1$}

In this case, $U \sim \zeta U_{1}+\zeta^{2} U_{2}+\cdots, V \sim V_{0}+\zeta V_{1}+$ $\cdots$, where $U_{1}, V_{0}$ are determined by (30) and (31), and $U_{2}$ is a solution of the next boundary problem

$$
\left\{\begin{array}{l}
\partial^{2} U_{2} / \partial \nu^{2}+2 i U_{2}=0,(\nu>0) \\
\frac{1}{\mu} \frac{\partial U_{2}}{\partial \nu}=-i \sin \vartheta U_{1}+\frac{\alpha}{\kappa_{0}} \frac{\partial U_{0}^{r}}{\partial n},(\nu=0)
\end{array} .\right.
$$

Hence

$$
\begin{aligned}
U_{2}(s, \nu) & =-\frac{i \mu U^{i}}{i-1}\left[\frac{i \alpha \bar{\kappa}}{\sin ^{2} \vartheta}+\frac{2 i \mu \sin ^{2} \vartheta}{i-1}\right] e^{(i-1) \nu} \\
V_{1}(s, \nu) & =-\frac{1}{i \mu} \frac{\partial U_{2}}{\partial \nu}=\frac{1-i}{i \mu} U_{2} .
\end{aligned}
$$

Analogous to (39), we may now write the condition on $S$ to consider finding the diffracted field in $G_{1}$ as the Dirichlet-type problem

$$
\begin{aligned}
& U^{r}=-U^{i}\{1-w {[2 \sin \vartheta} \\
&\left.\left.-\zeta\left(\frac{i \alpha \bar{\kappa}}{\sin ^{2} \vartheta}+\frac{2 i \mu \sin ^{2} \vartheta}{i-1}\right)+o(\zeta)\right]\right\} .
\end{aligned}
$$

As we see, the second asymptotic approximation meets the impedance condition (32). However, it can be shown that the 
third-order approximation $\left(U \sim \zeta U_{1}+\zeta^{2} U_{2}+\zeta^{3} U_{3}, V \sim\right.$ $\left.V_{0}+\zeta V_{1}+\zeta^{2} V_{2}\right)$ does not satisfy it. Indeed

$$
\left\{\begin{array}{l}
U_{3}(s, \nu)=\left[b_{0}+b_{1} /(i-1)-b_{1} \nu\right] e^{(i-1) \nu} \\
V_{2}(s, \nu)=\left[(1-i) U_{3}+b_{1} e^{(i-1) \nu}\right] /(i \mu)
\end{array}\right.
$$

where

$$
\begin{aligned}
& b_{0}=\frac{\mu^{2} U^{i}}{(i-1)^{2}}\left[-\frac{i \alpha \bar{\kappa}}{\sin \vartheta}-\frac{2 i \mu \sin ^{3} \vartheta}{i-1}+2 i \cos ^{2} \vartheta \frac{\partial \vartheta}{\partial \bar{s}}-2 i \bar{\kappa}\right] \\
& b_{1}=U^{i} \sin \vartheta i \mu\left[\left(\varepsilon_{1} \mu-\cos ^{2} \vartheta\right) /(i-1)+\alpha \bar{\kappa}\right] /(i-1) .
\end{aligned}
$$

Hence, the condition (32) is to be substituted by

$$
U+w V \sim-(1+i) b_{1}(s) \zeta^{3} / 2
$$

\section{Case $p>q$}

It is easy to derive from (19) and (16),

$$
\left\{\begin{array}{l}
U_{q+j}(s, \nu) \equiv 0 \quad(j=1,2, \ldots, q-1) \\
U_{2 q}(s, \nu)=i \mu^{2} U^{i} \sin ^{2} \vartheta^{(i-1) \nu} \\
V_{j}(s, \nu) \equiv 0 \quad(j=1,2, \ldots, q-1) \\
V_{q}(s, \nu)=(1-i) U_{2 q}(s, \nu) /(i \mu) .
\end{array}\right.
$$

Thus

$$
\begin{aligned}
& U \sim \zeta U_{q}(s, \nu)+\zeta^{2} U_{2 q}(s, \nu)+\cdots \\
& V \sim V_{0}(s, \nu)+\zeta V_{q}(s, \nu)+\cdots
\end{aligned}
$$

and curvature is not included in the first two terms of each expansion. The IBC is valid in the second-order approximation.

However, in the asymptotic approximation involving the term of $\zeta^{3}$ order for $U$ and the term of $\zeta^{2}$ order for $V$, the IBC (32) is not fulfilled.

For example, if $p=2, q=1$, then

$$
\begin{aligned}
& U \sim \zeta U_{1}+\zeta^{2} U_{2}+\zeta^{3} U_{3}+\cdots \\
& V \sim V_{0}+\zeta V_{1}+\zeta^{2} V_{2}+\cdots
\end{aligned}
$$

where $U_{1}, V_{0}, U_{2}, V_{1}$ are given by (30), (31), and (47), and

$$
\left\{\begin{array}{l}
U_{3}(s, \nu)=\left(c_{0}+c_{1} \nu\right) e^{(i-1) \nu} \\
V_{2}(s, \nu)=(1-i) U_{3}(s, \nu) /(i \mu)+i c_{1} e^{(i-1) \nu} / \mu
\end{array}\right.
$$

where

$$
\begin{aligned}
c_{0}= & c_{0}(s)=\frac{U^{i} \mu}{2(i-1)} \\
& \times\left[\mu^{2} \sin ^{3} \vartheta+\frac{2 \alpha \bar{\kappa}}{\sin ^{2} \vartheta}-\left(\varepsilon_{1} \mu-\cos ^{2} \vartheta\right) \sin \vartheta\right] \\
c_{1}= & c_{1}(s)=U^{i} \mu\left(\varepsilon_{1} \mu-\cos ^{2} \vartheta\right) \sin \vartheta / 2 .
\end{aligned}
$$

It follows from (50) that

$$
U+w V \sim(1+\mathrm{i}) c_{1}(s) \zeta^{3} / 2 \text { on } S
$$

and therefore the Leontovich condition (32) is to be substituted by (51).

It is obvious that for $p>2, q=1$ (and for any $p, q: p / q>2$ ) the third asymptotic term does not involve the curvature $\bar{\kappa}$. In general, the order of the curvature-corrected terms of the asymptotic expansion becomes higher, as the ratio $p / q$ gets bigger.

\section{Specialization to a Conducting Half-Space}

The exact closed-form solution of system (9), (16) can be easily obtained for a plane surface $S$ under condition (22)

$\tilde{U}(s, \nu)=2 U^{i} \mu e^{i \zeta \sqrt{\varepsilon \mu-\cos ^{2} \vartheta} \nu} /\left(\mu \sin \vartheta+\sqrt{\varepsilon \mu-\cos ^{2} \vartheta}\right)$.

If to expand it into series of $\zeta\left(\tilde{U}(s, \nu)=\sum_{m=1}^{\infty} \tilde{U}_{m}(s, \nu) \zeta^{m}\right)$, one can conclude that $\tilde{U}_{1}(s, 0)=2 U^{i} \sin \vartheta \mu /(1+i)$ is equal to $U_{q}(s, 0)$ in $(30)$, and the next coefficients

$$
\begin{aligned}
& \tilde{U}_{2}(s, 0)=U^{i} \sin ^{2} \vartheta i \mu^{2} \\
& \tilde{U}_{3}(s, 0)=-\frac{U^{i} \sin \vartheta \mu \sqrt{\varepsilon_{1} \mu-\cos ^{2} \vartheta}}{2(i-1)}
\end{aligned}
$$

are incorporated into (42), (45) or (47), (50).

Thus, the principal term of asymptotic expansion (20) is "pure" geometrical optics, while the subsequent terms provide correction to it.

\section{H-POLARIZATION}

Since the method and solution of the problem in this case do not differ much from those for E-polarization, we present here just the final formulas.

When $p<q$, we have

$U=U_{0}+\zeta^{\frac{p}{q}} U_{p}+o\left(\zeta^{\frac{p}{q}}\right) ; \quad V=\zeta V_{q}+\zeta^{\frac{p+q}{q}} V_{p+q}+o\left(\zeta^{\frac{p+q}{q}}\right)$

where

$$
\left\{\begin{array}{l}
U_{0}(s, \nu)=2 U^{i} e^{(i-1) \nu}, U_{p}(s, \nu)=i \alpha \bar{\kappa} U^{i} e^{(i-1) \nu} / \sin ^{3} \vartheta \\
V_{q}=(i-1) \mu U_{0} / 2, V_{p+q}=(i-1) \mu U_{p} / 2
\end{array}\right.
$$

and, hence, in the second-order approximation

$$
V \sim-w U
$$

On the surface $S$ the diffracted field $U^{r}$ satisfies the following condition:

$$
\left.U^{r}\right|_{S}=U^{i}\left[1+\zeta^{p / q} i \alpha \bar{\kappa} / \sin ^{3} \vartheta+o\left(\zeta^{p / q}\right)\right]
$$

which can be used instead of (55).

Same as for E-polarization, impedance condition (55) is violated in the asymptotic approximation comprising terms of $\zeta^{(p+q) / q}$ order.

$$
\text { If } p=q=1 \text {, then }
$$

$$
U=U_{0}+\zeta U_{1}+o(\zeta), \quad V=\zeta V_{1}+\zeta^{2} V_{2}+o\left(\zeta^{2}\right)
$$


where

$$
\left\{\begin{array}{l}
U_{0}=2 U^{i} e^{(i-1) \nu}, U_{1}=U_{0}\left[\frac{i \alpha \bar{\kappa}}{\sin ^{3} \vartheta}+\frac{\mu(i-1)}{\sin \vartheta}\right] e^{(i-1) \nu} \\
V_{1}=\mu(i-1) U_{0} / 2, V_{2}=\mu(i-1) U_{1} / 2
\end{array}\right.
$$

and further conclusions are formulated similarly to $p<q$.

Let now $p>q$. Then

$$
U=U_{0}+\zeta U_{q}+o(\zeta), \quad V=\zeta V_{q}+\zeta^{2} V_{2 q}+o\left(\zeta^{2}\right)
$$

Here

$$
\left\{\begin{array}{l}
U_{0}=2 U^{i} e^{(i-1) \nu}, U_{q}=(i-1) \mu U^{i} e^{(i-1) \nu} / \sin \vartheta \\
V_{q}=(i-1) \mu U_{0} / 2, V_{2 q}=(i-1) \mu U_{q} / 2 .
\end{array}\right.
$$

The second-order approximation, as we see, satisfies Leontovich condition (55), but does not comprise the curvature, unlike the case $p \leq q$.

Let us find curvature-corrected terms in particular case of $p=$ $2, q=1$

$U=U_{0}+\zeta U_{1}+\zeta^{2} U_{2}+o\left(\zeta^{2}\right), V=\zeta V_{1}+\zeta^{2} V_{2}+\zeta^{2} V_{3}+o\left(\zeta^{3}\right)$

where $U_{0}, U_{1}, V_{1}, V_{2}$ are expressed by (60) for $q=1$, and

$$
U_{2}(s, \nu)=\left[\tilde{c}_{0}(s)+\nu \tilde{c}_{1}(s)\right] e^{(i-1) \nu}
$$

where

$$
\begin{aligned}
& \tilde{c}_{0}(s)=i U^{i}\left(\alpha \bar{\kappa}-\mu^{2} \sin ^{2} \vartheta\right) / \sin ^{3} \vartheta \\
& \tilde{c}_{1}(s)=U^{i}(1+i)\left(\varepsilon_{1} \mu-\cos ^{2} \vartheta\right) / 2 .
\end{aligned}
$$

From (11) follows:

$$
\begin{aligned}
V_{3}=\frac{\mu}{2} \frac{\partial U_{2}}{\partial \nu}+ & \frac{i \varepsilon_{1} \mu^{2}}{4} \frac{\partial U_{0}}{\partial \nu}=-\frac{\mu(1-i)}{2} U_{2}(s, \nu) \\
& -\mu(1+i) U^{i}\left(\varepsilon_{1} \mu+\cos ^{2} \vartheta\right) e^{(i-1) \nu} / 4
\end{aligned}
$$

Consequently, in the third-order approximation

$$
V \sim-w U-\mu(1+i) \zeta_{1}^{3} U^{i}\left(\varepsilon_{1} \mu+\cos ^{2} \vartheta\right) / 4, \quad(\nu=0) .
$$

Therefore homogeneous impedance condition (55) is to be replaced by (63) (or by its Dirichlet-type equivalent).

\section{VERIFICATION METHOD AND NUMERICAL VALIDATION}

The verification was performed by solving Muller-type boundary integral equations (MBIEs) [24]

$$
\begin{aligned}
& U(\vec{x})+\frac{1}{2 \pi} \int_{S} K_{11}(\vec{x}, \vec{y}) U(\vec{y}) d s_{y} \\
& -\frac{k_{0}}{2 \pi} \int_{S} K_{12}(\vec{x}, \vec{y}) V(\vec{y}) d s_{y}=U^{i}(\vec{x}) \\
& \frac{k_{0}}{2}\left(1+p_{i}\right) V(\vec{x})+\frac{1}{2 \pi} \int_{S} K_{21}(\vec{x}, \vec{y}) U(\vec{y}) d s_{y} \\
& -\frac{1}{2 \pi} \int_{S} K_{22} V(\vec{y}) d s_{y}=\frac{\partial U^{i}(\vec{x})}{\partial n_{x}} \quad \vec{x} \in S
\end{aligned}
$$

where

$$
\begin{aligned}
k_{0} V(\vec{x}) & =\partial U(\vec{x}) / \partial n_{x}, k_{0} V(\vec{y})=\partial U(\vec{y}) / \partial n_{y} \\
K_{11} & =\partial G_{i}(\vec{x}, \vec{y}) / \partial n_{y}-\partial G_{e}(\vec{x}, \vec{y}) / \partial n_{y} \\
K_{12} & =G_{i}(\vec{x}, \vec{y})-p_{i} G_{e}(\vec{x}, \vec{y}) \\
K_{21} & =\partial^{2} G_{i}(\vec{x}, \vec{y}) / \partial n_{x} \partial n_{y}-\partial^{2} G_{e}(\vec{x}, \vec{y}) / \partial n_{x} \partial n_{y} \\
K_{22} & =\partial G_{i}(\vec{x}, \vec{y}) / \partial n_{x}-p_{i} \partial G_{e}(\vec{x}, \vec{y}) / \partial n_{x}
\end{aligned}
$$

and $G_{i, e}=i \pi / 2 H_{0}^{(1)}\left(k_{i, 0} r\right)$ are the solutions of Helmholz equation in the space with parameters $\varepsilon, \mu$ and in the free-space, respectively, and $r=|\vec{x}-\vec{y}|$. Constant $p_{i}=\mu$ in the E-polarization case, and $p_{i}=\varepsilon$ for the H-polarization.

As kernels of the MBIEs have at most a weak logarithmic singularity, and the MBIEs are free of false resonances [24], we are free in choosing numeric scheme of their discretization. In our consideration we are using piecewise-constant approximation of $U, V$ after subtraction and analytical integration of the logarithmic singularity.

In order to estimate the accuracy of our asymptotic approximations, we compare it with numerical solutions of MBIEs. Since the accuracy of a fixed asymptotic order turns out to be the same for both polarizations, here we present only data for the E-polarization case. Figs. 2 and 3 illustrate the magnetic current relative error in the E-polarization case of plane wave diffraction by a circular conducting cylinder

$$
\Delta=\frac{\| V^{\mathrm{AS}}|-| V^{\mathrm{IE}}||}{\left|V^{\mathrm{IE}}\right|} \cdot 100 \%
$$

where superscripts "AS", "IE" refer to the asymptotic and the MBIEs' solutions, respectively. Since field computation on the boundary by asymptotic techniques has a local character, the better sample for verification is not an object of complex geometry, but a circular cylinder, as it allows to estimate contribution of the main factors: the boundary curvature and the tangent slope. For more complex geometries with significantly changing curvature of a boundary, asymptotic expansions for different $p, q$ can be used in different regions of the surface.

Our goal was to analyze the necessity of applying higherorder boundary conditions to different types of materials and different ratios between $\eta$ and $\zeta$. Series of computations showed that good coincidence of the data computed by two methods occurs, when the surface maximal curvature radius $R>0.7 \lambda$. A good accuracy for minimal sizes and curvatures of a scatterer is demonstrated by Figs. 2-4.

As for metals parameter $\zeta$ is relatively small (Fig. 2), the accuracy achieved by a second term of asymptotic expansion is not corrected significantly by the higher-order approximations. Compared to zero-order approximation, the first one is applicable for wider range of $\vartheta$ values: the relative error less than $5 \%$ is observed for all $\vartheta<65^{\circ}$. The same degree of accuracy is observed for other types of metals (copper, silver etc.).

Similar results are obtained for a type of biological tissue, whose conductivity is $\sigma=1.8 \cdot 10^{11} \mathrm{sec}^{-1}$ [21] (Fig. 3). (We recall that all conductivity values are given in Gaussian unit system). However, in this case ( $p=2, q=1)$ zero-order approximation rises up to $13 \%$, and to achieve acceptable accuracy 


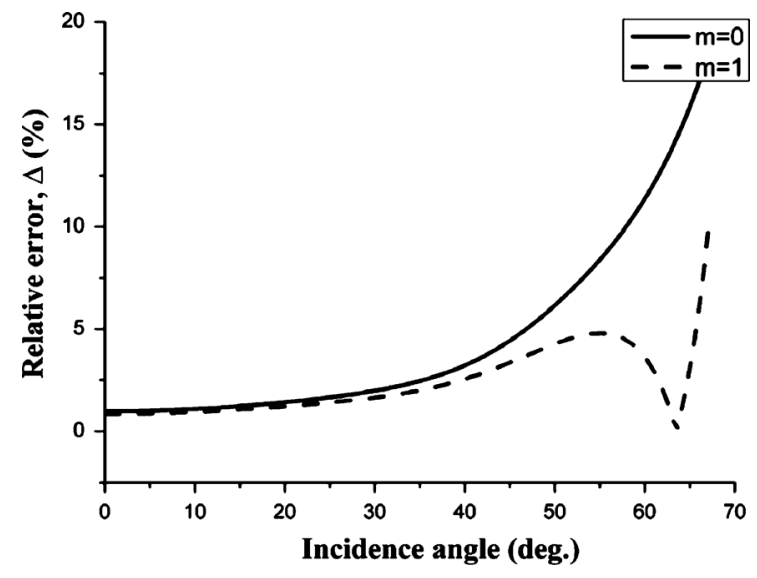

Fig. 2. Relative error of the first and the second term approximations of $|\mathrm{V}|$ compared to IE solution, for a stainless steel $\left(\sigma=1.3 \cdot 10^{16} \mathrm{sec}^{-1}\right)$ circular cylinder and parameters: $\lambda=1 \mathrm{~cm}, R_{0}=1.5 \lambda,(p=1, q=2, \eta=0.106$, $\zeta=0.0015, \alpha=2.75)$.

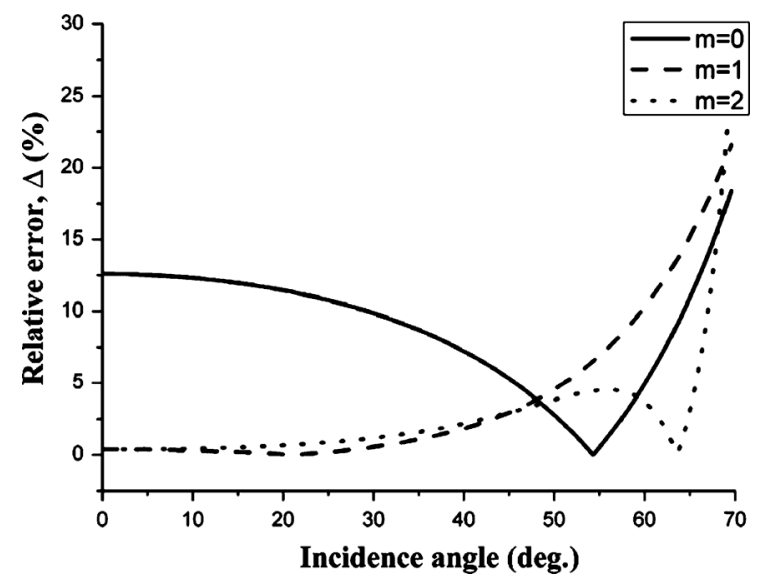

Fig. 3. Same as in Fig. 2, but for $R_{0}=\lambda\left(\varepsilon_{1}=40, \sigma=1.8 \cdot 10^{11} \mathrm{sec}^{-1}, p=\right.$ $2, q=1, \lambda=3 \mathrm{~cm}, \eta=0.159, \zeta=0.23, \alpha=2.96)$.

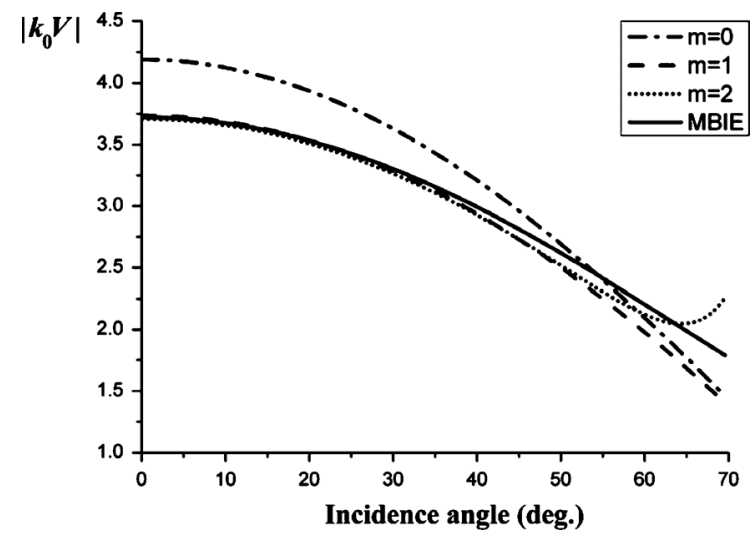

Fig. 4. Absolute value of $k_{0} V$ for parameters of Fig. 3.

in the wide range of surface slope angles, the second-order approximation is to be employed. Note that it already comprises corrections (44) to Leontovich condition. The absolute values of the fields in the higher-order approximations are given in Fig. 4.

\section{CONCLUSION}

The asymptotic formulas derived in the paper are applicable to high-frequency diffraction by a slightly curved conducting body. They lead to Dirichlet boundary condition, which has clear advantages compared to the IBC. The accuracy of IBC decreases for materials with smaller conductivities. Thus, the higher-order corrections to the IBC are required in this case. The curvature-corrected higher-order terms of the asymptotic expansion can also be used for bodies, whose local curvature radii are compared to the wavelength.

\section{REFERENCES}

[1] M. A. Leontovich, "On approximate boundary conditions for electromagnetic fields on the surface of highly conducting bodies," Res. Propag. Radio Waves, pp. 5-12, 1948, part. II, Moscow, (in Russian).

[2] O. I. Panych, "On the approximate boundary conditions," Doklady Akad. Nauk SSSR, vol. 20, no. 4, pp. 589-592, 1950.

[3] T. B. A. Senior, "Impedance boundary conditions for imperfectly conducting surfaces," Appl. Sci. Res. B., vol. 8, pp. 418-436, 1960.

[4] H. Haddar, P. Joly, and H.-M. Nguyen, "Generalized impedance boundary conditions for scattering problems from strongly absorbing obstacles: The case of Maxwell's equations," Math. Models Meth. Appl. Sci., vol. 18, no. 10, pp. 1787-1827, 2008.

[5] T. B. A. Senior and J. L. Volakis, "Approximate boundary conditions," IEE Electromagn. Waves Theories, vol. 41, 1995.

[6] D. S. Wang, "Limits and validity of the impedance boundary condition on penetrable surfaces," IEEE Trans. Antennas Propag., vol. 35, no. 4, pp. 453-457, Apr. 1987.

[7] S. Yuferev and L. Di Rienzo, "Surface impedance boundary conditions in terms of various formalisms," IEEE Trans. Magn., vol. 46, no. 9, pp. 3617-3628, Sep. 2010.

[8] I. V. Sukharevskii, "Passage of electromagnetic waves through a radiotransparent layer," Radio Eng. Electron. Phys., vol. 12, pp. 191-197, 1967.

[9] I. V. Sukharevsky, "On the influence of curvature and non-equidistance of boundaries to the passage of waves through a dielectric layer," in All-Union Symp. Diffraction and Propagation of Waves (in Russian), Kharkov, Ukraine, 1967, Abstracts.

[10] E. N. Semeniaka, "Asymptotic solution of the problem of diffraction of electromagnetic waves from a slightly bent dielectric layer of much greater thickness than the wavelength," Radio Eng. Electron. Phys., vol. 18 , pp. $41-49,1973$.

[11] E. N. Semenyaka and I. V. Sukharevsky, "Diffraction by slightly curved layered structures of big thickness," in Proc. V All-Union Symp. Diffraction Propag. Waves (in Russian), Leningrad, USSR, Jul. 13-17, 1970, pp. 118-124.

[12] V. I. Zamiatin, "The diffraction of electromagnetic waves by radiotransparent layers of finite thickness," Radio Eng. Electron. Phys., vol. 19, pp. 5-12, 1974.

[13] Yu. G. Gukasov and I. V. Sukharevsky, "Asymptotic treatment of short-wave radiation of dielectric-coated reflector antenna," Radio Eng. Electron. Phys., no. 5, pp. 784-790, 1963.

[14] S. I. Grinberg, E. N. Semenyaka, and I. V. Sukharevskii, "Short-wave asymptotic behaviour of Green's function in the problem of diffraction at a plane layer," USSR Comput. Math. Math. Phys., vol. 13, no. 3, pp. 170-186, 1973

[15] A. G. Kravchenko and I. V. Sukharevsky, "Asymptotic solution of one class of short-wave diffraction problems for bodies with big (finite) conductivity," in Proc. V All-Union Symp. on Diffraction and Propagation of Waves (in Russian), Leningrad, USSR, Jul. 13-17, 1970, pp. $118-124$.

[16] A. G. Kravchenko, "On the high-frequency diffraction by good conductors," Radiotekh. Electron., vol. XVIII, no. 1, pp. 49-57, in Russian.

[17] A. G. Kravchenko, "Scattering by a body described by the model of Zommerfeld," in Proc. VI All-Union Symp. on Diffraction and Propagation of Waves (in Russian), Moscow-Yerevan, 1973, pp. 368-372.

[18] S. M. Rytov, "Calcul du skin-effet par la méthode des perturbations," J. Phys. USSR, vol. T.2., no. 3, pp. 233-242, 1940.

[19] S. V. Yuferev and N. Ida, Surface Impedance Boundary Conditions. Boca Raton, FL, USA: CRC, 2010. 
[20] E. Bleszynski, M. Bleszynski, and T. Jaroszewicz, "Surface-integral equations for electromagnetic scattering from impenetrable and penetrable sheets," IEEE Antennas Propag. Mag., vol. 36, no. 2, pp. 14-25, 1993.

[21] C. Gabriel, S. Gabriel, and E. Corthout, "The dielectric properties of biological tissues: I. Literature," Phys. Med. Biol., vol. 41, pp. 2231-2249, 1996

[22] J. E. Hipp, "Soil electromagnetic parameters as functions of frequency, soil density, and soil moisture," Proc. IEEE, vol. 62, no. 1, pp. 98-103, Jan. 1974

[23] D. A. McNamara, C. W. I. Pistorius, and J. A. G. Malherbe, The Uniform Geometrical Theory of Diffraction. London, U.K.: Artech House, 1990.

[24] M. V. Balaban, E. I. Smotrova, O. V. Shapoval, V. S. Bulygin, and A. I. Nosich, "Nystrom-type techniques for solving electromagnetics integral equations with smooth and singular kernels," Int. J. Numer. Model.: Electronic Netw., Devices Fields, vol. 25, no. 5, pp. 490-511, 2012.

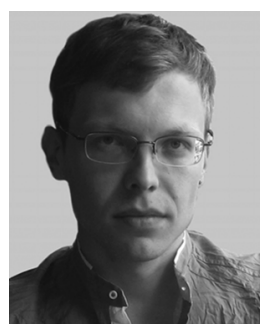

Ilya O. Sukharevsky (S'08-M'13) was born in Kharkov, Ukraine, in 1984. He received the B.Sc. and the M.Sc. degrees in mathematics from Kharkov National University, Ukraine, and the Ph.D. from the Institute for Radiophysics and Electronics of the National Academy of Sciences of Ukraine (NASU), Kharkov, Ukraine, in 2005, 2006, and 2011, respectively.

$\mathrm{He}$ is currently doing postdoctoral research at the Communication and Spectrum Management Research Center (ISYAM), Bilkent University, Ankara, Turkey. His scientific interests are in the integral equation technique and asymptotic methods of electromagnetics.

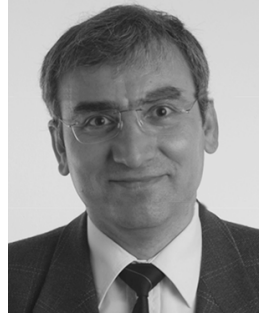

Ayhan Altintas (SM'93) received the B.S. and M.S. degrees from the Middle East Technical University, Ankara, Turkey, and the Ph.D. degree from The Ohio State University, Columbus, OH, USA, in 1979,1981 , and 1986, respectively, all in electrical engineering.

Currently, he is a Professor of electrical engineering at Bilkent University, Ankara, Turkey, and the Faculty of Engineering and Natural Sciences, Abdullah Gul University, Kayseri, Turkey. He is also the Director of Communication and Spectrum Management Research Center (ISYAM). His research interests are in electromagnetics, antennas, propagation, and wireless communication systems.

Prof. Altintas was Chairman of the IEEE Turkey Section in 1991-1993 and 1995-1997. He is the Founder and First Chair of the IEEE AP/MTT Chapter in the Turkey Section. At present, he is the President of URSI Turkish National Committee. He is a Fulbright Scholar and an Alexander von Humboldt Fellow. He received The Ohio State University ElectroScience Laboratory Outstanding Dissertation Award of 1986, the IEEE 1991 Outstanding Student Branch Counselor Award, the 1991 Research Award of the Prof. M. N. Parlar foundation of METU, and the Young Scientist Award of the Scientific and Technical Research Council of Turkey in 1996. He is a member of Sigma Xi and Phi Kappa Phi and a recipient of IEEE Third Millennium Medal. 